\title{
Dirac neutrino mass generation from a Majorana messenger
}

\author{
Julian Calle๑, ${ }^{1, *}$ Diego Restrepo $\odot,{ }^{1,2, \uparrow}$ and Óscar Zapata $\odot^{1,3, *}$ \\ ${ }^{1}$ Instituto de Física, Universidad de Antioquia, \\ Calle 70 No. 52-21, Apartado Aéreo 1226, Medellín, Colombia \\ ${ }^{2}$ International Institute of Physics, Universidade Federal do Rio Grande do Norte, \\ Campus Universitario, Lagoa Nova, Natal-RN 59078-970, Brazil \\ ${ }^{3}$ Abdus Salam International Centre for Theoretical Physics, Strada Costiera 11, 34151, Trieste, Italy
}

(Received 29 October 2019; accepted 19 December 2019; published 6 February 2020)

\begin{abstract}
The radiative type-I seesaw has been already implemented to explain the lightness of Majorana neutrinos with both Majorana and Dirac heavy fermions, and the lightness of Dirac neutrinos with Dirac heavy fermions. In this work, we present a minimal implementation of the radiative type-I seesaw with light Dirac neutrinos and heavy Majorana fermions. An inert doublet and a complex singlet scalar complete the dark sector, which is protected by an Abelian fermiophobic gauge symmetry that also forbids tree level mass contributions for the full set of light neutrinos. A fermion vectorlike extension of the model is also proposed where the light right-handed neutrinos can thermalize in the primordial plasma and the extra gauge boson can be directly produced at colliders. In particular, the current upper bound on $\Delta N_{\text {eff }}$ reported by PLANCK points to large ratios of $M_{Z^{\prime}} / g^{\prime} \gtrsim 40 \mathrm{TeV}$, which can be competitive with collider constraint for $g^{\prime}$ sufficiently large in the ballpark of the Standard Model values, while future cosmic microwave background experiments may probe all the no minimal models presented here.
\end{abstract}

DOI: 10.1103/PhysRevD.101.035004

\section{INTRODUCTION}

The interpretation of neutrino experimental data in terms of neutrino oscillations is compatible with both Majorana and Dirac neutrino masses [1]. The former possibility has received the most attention but given the lack of signals in neutrinoless double beta decay experiments [2-7], the latter cannot be dismissed. If neutrinos are Dirac particles, the Standard Model (SM) particle content must be extended with right-handed neutrinos, which can increase the effective number of light neutrinos, $N_{\text {eff }}$, until six. Therefore, to be compatible with the current cosmological restrictions on $N_{\text {eff }}$, the interactions of the extra right-handed neutrinos with the primordial plasma must be highly suppressed.

On the other hand, to give small masses to at least two Majorana or Dirac neutrinos, as required to explain the neutrino oscillation experiments $[8,9]$, the seesaw mechanism with heavy fermions is usually invoked. For the treelevel type-I seesaw, we can have either light Majorana neutrinos with heavy Majorana mediators [10-13] or light

\footnotetext{
julian.callem@udea.edu.co restrepo@udea.edu.co

¥oalberto.zapata@udea.edu.co
}

Published by the American Physical Society under the terms of the Creative Commons Attribution 4.0 International license. Further distribution of this work must maintain attribution to the author(s) and the published article's title, journal citation, and DOI. Funded by SCOAP ${ }^{3}$.
Dirac neutrinos with heavy Dirac mediators [14-17]. The radiative type-I seesaw includes both [18] possibilities [19], but now it is also possible to have light Majorana neutrinos with heavy Dirac mediators [20]. In this work, we want to explore the possibility to build a simple Dirac radiative type-I seesaw model with heavy Majorana mediators. It is worth noticing that this idea have been already illustrated in an extension of the minimal supersymmetric standard model [21] but without show any explicit solution.

In general, solutions for light Dirac neutrino masses require a continuous symmetry to guarantee their Diracness. This symmetry is usually identified as the local $\mathrm{U}(1)_{B-L}$. Additionally, ad hoc discrete symmetries are invoked to forbid tree level Dirac or Majorana mass terms for the light right-handed neutrinos [14,22,23]. However, tree-level Dirac type-I seesaw with proper choices for the $\mathrm{U}(1)_{B-L}$ charges have been shown to be consistent without requiring any extra ad hoc discrete symmetries [17]. In recent works, it has been shown that even for one-loop Dirac neutrino masses, it is possible to have $\mathrm{U}(1)_{B-L}$ as the only extra symmetry beyond the SM [24-26]. ${ }^{\circ}$

As a bonus in this case, the new scalars and fermions circulating the loop can be dark matter candidates with the stability of the lightest of them guaranteed by the very same continuous symmetry. We focus here on solutions for the

\footnotetext{
${ }^{1}$ For extensions with only extra scalars, minimal solutions have been found with two and three loops [26].
} 
radiative Dirac type-I seesaw with Majorana mediators, which have only an extra local symmetry responsible for the Diracness of the light neutrinos, the absence of any treelevel mass, and the existence of a dark sector constituted by the particles circulating the loop.

In fact, another evidence that the SM is not a complete theory is the missing matter content of the Universe, which is known as dark matter (DM). The main proposals that explain DM as a particle are given in Ref. [27]. However, there has been only gravitational evidence for the existence of dark matter so far. Without evidence of DM as a particle, there is not a clear path to pin out the DM properties nor the possible heavier companions of some extended dark sector. Linking the dark sector to other specific phenomenology allows us to reduce the arbitrariness in the model building. In our construction, the dark sector is related to the heavy sector responsible of the lightness of the neutrinos, and the same symmetry that guarantees the lightness of the Dirac neutrinos is the responsible of the stability of the lightest dark particle (LDP). Therefore, the number of specific models is quite restricted.

The rest of the paper is organized as follows. In the next section, we present the model and study the scalar mass spectrum after spontaneous symmetry breaking. In Sec. III, we present the radiative mechanism that generates Dirac neutrino masses and establish the lepton flavor violation constraints. The different resulting DM scenarios are discussed in Sec. IV, and in Sec. V, we show the cosmological restrictions $\left(N_{\text {eff }}\right)$ in a nonminimum model for different extra Abelian symmetries.

\section{THE MODEL}

We extend the SM with a spontaneously broken Abelian gauge symmetry which guarantees the Dirac-ness of the massive neutrinos. Only the new particles, including the right-handed partners of the SM neutrinos, are charged under this new $\mathrm{U}(1)_{\mathcal{D}}$ dark gauge symmetry [28-30] to obtain an anomaly free theory. We choose the new particle set such that the following dimension six operator is realized at one-loop level,

$$
\mathcal{O}_{6 D}=\frac{1}{\Lambda^{2}} \bar{L} \tilde{H} \nu_{R} S^{2}
$$

where $S$ is the singlet scalar field which spontaneously breaks the $\mathrm{U}(1)_{\mathcal{D}}$ symmetry needed to forbid the Dirac and Majorana neutrino mass terms at tree level.

With the aim to illustrate the one-loop Dirac neutrino mass generation, we consider the particle content shown in Table I as a possible realization of the effective operator $\mathcal{O}_{6 D}$. Specifically, we introduce three scalar fields $\eta, \sigma$, and $S$, where only $S$ develops a nonzero vacuum expectation value (VEV), a set of three singlet fermions, $\nu_{R j}(j=1,2)$ and $\nu_{R 3}$, and another set of three heavy Majorana fermions, $\psi_{R \alpha}(\alpha=1,2,3)$. The $\mathrm{U}(1)_{\mathcal{D}}$ charges for the new particles
TABLE I. The new scalars and fermions with their respective charges. All the SM fields are neutral under the dark $\mathrm{U}(1)_{\mathcal{D}}$ gauge symmetry.

\begin{tabular}{lccc}
\hline \hline Fields & $\mathrm{SU}(2)_{L}$ & $\mathrm{U}(1)_{Y}$ & $\mathrm{U}(1)_{\mathcal{D}}$ \\
\hline$\eta$ & $\mathbf{2}$ & 1 & 1 \\
$S$ & $\mathbf{1}$ & 0 & 2 \\
$\sigma$ & $\mathbf{1}$ & 0 & 3 \\
$\nu_{R i}$ & $\mathbf{1}$ & 0 & -4 \\
$\nu_{R 3}$ & $\mathbf{1}$ & 0 & 5 \\
$\psi_{R \alpha}$ & $\mathbf{1}$ & 0 & 1 \\
\hline \hline
\end{tabular}

are defined by the anomaly cancellation conditions and the gauge invariance in Yukawa and scalar interactions.

The most general Lagrangian for some gauge $\mathrm{U}(1)_{X}$ symmetry, which includes the trivial case $X=\mathcal{D}$, must contains the following gauge, Yukawa, and scalar interactions in order to realize $\mathcal{O}_{6 D}$ at one-loop:

$$
\begin{aligned}
\mathcal{L} \supset & -g^{\prime} Z_{\mu}^{\prime} \sum_{F} q_{F} \bar{F} \gamma^{\mu} F+\sum_{\phi}\left|\left(\partial_{\mu}+i g^{\prime} q_{\phi} Z_{\mu}^{\prime}\right) \phi\right|^{2} \\
& -\left[h_{i \alpha} \overline{L_{i}} \tilde{\eta} \psi_{R \alpha}+y_{j \alpha} \overline{\nu_{R_{j}}} \sigma^{*} \psi_{R \alpha}^{c}+\kappa_{\alpha \beta} \overline{\psi_{R \alpha}^{c}} \psi_{R \beta} S^{*}+\text { H.c. }\right] \\
& -\mathcal{V}(H, S, \eta, \sigma) .
\end{aligned}
$$

In the first row, $g^{\prime}$ is the gauge coupling associated to the $\mathrm{U}(1)_{X}$ group and $Z_{\mu}^{\prime}$ is its corresponding gauge boson, $F$ $(\phi)$ denote the new fermions (scalars), and $q_{F, \phi}$ their $X$ charges. In the second row, $L_{i}(i=1,2,3)$ and $H$ are the SM lepton and Higgs doublets, respectively, $\tilde{\eta}=i \sigma_{2} \eta^{*}$, and $h, y$, and $\kappa$ are matrices in the flavor space. The scalar potential can be cast as

$$
\begin{aligned}
\mathcal{V}(H, S, \eta, \sigma)= & V(H)+V(S)+V(\eta)+V(\sigma) \\
& +\lambda_{1}\left(H^{\dagger} H\right)\left(S^{*} S\right)+\lambda_{2}\left(H^{\dagger} H\right)\left(\sigma^{*} \sigma\right) \\
& +\lambda_{3}\left(H^{\dagger} H\right)\left(\eta^{\dagger} \eta\right)+\lambda_{4}\left(S^{*} S\right)\left(\sigma^{*} \sigma\right) \\
& +\lambda_{5}\left(S^{*} S\right)\left(\eta^{\dagger} \eta\right)+\lambda_{6}\left(\eta^{\dagger} \eta\right)\left(\sigma^{*} \sigma\right) \\
& +\lambda_{7}\left(\eta^{\dagger} H\right)\left(H^{\dagger} \eta\right)+\lambda_{8}\left(\eta^{\dagger} H S^{*} \sigma+\text { H.c. }\right),
\end{aligned}
$$

with $V(\omega)=\mu_{\omega}^{2} \omega^{\dagger} \omega+\lambda_{\omega}\left(\omega^{\dagger} \omega\right)^{2}$. It is worth to emphasize that $\mathrm{U}(1)_{\mathcal{D}}$ automatically allows for all the terms in Eqs. (2) and (3), but other realizations will be checked later on. Note that after the spontaneous symmetry breaking of $\mathrm{U}(1)_{X}$, the $\lambda_{8}$ term gives rise to the mixing between the neutral parts of $\eta$ and $\sigma$, which is mandatory to generate nonzero radiative masses. We assume $\lambda_{8}$ and $\langle S\rangle$ reals to preserve the $C P$ symmetry in the scalar sector, and $\mu_{\eta}^{2}, \mu_{\sigma}^{2}>0$ to avoid treelevel mixing terms among the fermions. Moreover, we also assume $\lambda_{1} \ll 1$ such that the scalar $S$ and $H$ do not mix allowing us to identify the $C P$ even scalar particle in $H$ as the SM Higgs boson. To establish the scalar spectrum, we expand the scalar fields as 


$$
\begin{aligned}
& H=\left(\begin{array}{c}
G^{+} \\
\frac{1}{\sqrt{2}}\left(h+v_{H}+i G\right)
\end{array}\right), \quad \eta=\left(\begin{array}{c}
\eta^{+} \\
\frac{1}{\sqrt{2}}\left(\eta_{R}+i \eta_{I}\right)
\end{array}\right), \\
& S=\frac{1}{\sqrt{2}}\left(S_{R}+v_{S}+i S_{I}\right), \quad \sigma=\frac{1}{\sqrt{2}}\left(\sigma_{R}+i \sigma_{I}\right),
\end{aligned}
$$

with $v_{H}=246.22 \mathrm{GeV}$. Of the original 12 scalar degrees of freedom (d.o.f.) in the model, the gauge bosons $W^{ \pm}, Z^{0}$, and $Z^{\prime}$ absorb four of them (the Goldstone bosons $G^{ \pm}, G$, and $S_{I}$ ). Thus, the scalar spectrum contains two sets of two neutral $C P$-even states ( $h$ and $S_{R}$, and $\sigma_{R}$ and $\eta_{R}$ ), two $C P$ odd scalar states $\left(\sigma_{I}\right.$ and $\left.\eta_{I}\right)$, and one charged scalar $\left(\eta^{ \pm}\right)$. The mass spectrum for the unmixed scalars reads

$$
\begin{aligned}
& m_{\eta^{ \pm}}^{2}=\mu_{\eta}^{2}+\frac{1}{2}\left(\lambda_{3} v_{H}^{2}+\lambda_{5} v_{S}^{2}\right), \\
& m_{H}^{2}=\lambda_{H} v_{H}^{2}, \quad m_{S}^{2}=\lambda_{S} v_{S}^{2} .
\end{aligned}
$$

The other mass eigenstates are defined as

$$
\left(\begin{array}{l}
\chi_{(R, I)_{1}} \\
\chi_{(R, I)_{2}}
\end{array}\right)=\left(\begin{array}{cc}
\cos \theta & -\sin \theta \\
\sin \theta & \cos \theta
\end{array}\right)\left(\begin{array}{l}
\sigma_{(R, I)} \\
\eta_{(R, I)}
\end{array}\right),
$$

where $\tan \theta=2 c /(b-a)$, with $a=m_{\eta^{ \pm}}^{2}+\frac{1}{2} \lambda_{7} v_{H}^{2}, b=\mu_{\sigma}^{2}+$ $\frac{1}{2}\left(\lambda_{2} v_{H}^{2}+\lambda_{4} v_{S}^{2}\right)$, and $c=\frac{1}{2} \lambda_{8} v_{H} v_{S}$. Note that the $C P$-even states $\chi_{R(1,2)}$ are mass degenerate with the $C P$-odd ones $\chi_{I(1,2)}$, with masses $m_{\chi_{(1,2)}^{2}}^{2}=\left[a+b \mp \sqrt{(a-b)^{2}+4 c^{2}}\right] / 2$.

On the other hand, we assume that the heavy Majorana fermions are already in the diagonal basis in such a way their masses are $M_{\psi_{\alpha}}=\kappa_{\alpha \alpha} v_{S} / \sqrt{2}$, with $M_{\psi_{1}}<M_{\psi_{2}}<M_{\psi_{3}}$. Finally, the mass of the new gauge boson is given by

$$
M_{Z^{\prime}}=q_{S} g^{\prime} v_{S}
$$

\section{NEUTRINO MASSES AND CHARGED LEPTON FLAVOR VIOLATION}

Neutrino masses are generated at one-loop level according to the diagram in Fig. 1. The expression for the effective neutrino mass matrix $\mathcal{M}_{\nu}$ can be cast as

$$
\begin{aligned}
\left(\mathcal{M}_{\nu}\right)_{i j}= & \frac{1}{32 \pi^{2}} \frac{\lambda_{8} v_{S} v_{H}}{m_{\chi_{R_{2}}}^{2}-m_{\chi_{R_{1}}}^{2}} \sum_{\alpha=1}^{3} h_{i \alpha} M_{\psi_{\alpha}} y_{j \alpha}^{*} \\
& \times\left[F\left(\frac{m_{\chi_{R_{2}}}^{2}}{M_{\psi_{\alpha}}^{2}}\right)-F\left(\frac{m_{\chi_{R_{1}}}^{2}}{M_{\psi_{\alpha}}^{2}}\right)\right]+(R \rightarrow I),
\end{aligned}
$$

where $F(x)=x \log x /(x-1)$. Note that the structure of the effective neutrino mass matrix, given by the product $\left(M_{\nu}\right)_{i j} \propto h_{i \alpha} y_{j \alpha}$, is similar to the structure of the neutrino mass matrix for the tree-level seesaw mechanism for Dirac neutrinos [31]. It is also worth mentioning that if only one fermion $\psi_{R}$ is added, then there will be two massless

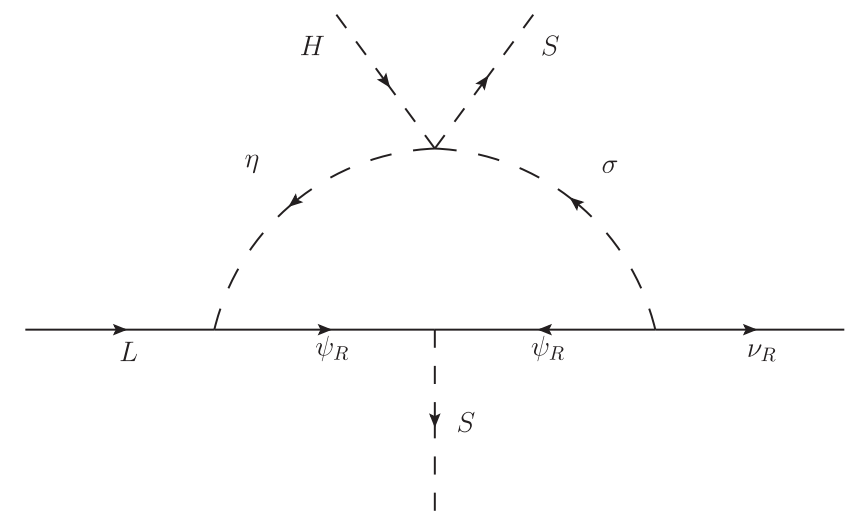

FIG. 1. One-loop realization of the dimension-6 operator $\bar{L} \tilde{H} \nu_{R} S^{2}$ leading to Dirac neutrino masses with Majorana mediators.

neutrinos, which would be ruled out by the current neutrino oscillation data [32]. In our case, we assume the existence of three of such fermions, generating Dirac scotogenic masses for the two left-handed neutrinos $\left(\nu_{3}=\nu_{L 3}+\nu_{R 3}\right.$ is massless due to the charge assignment).

In order to estimate the possible values for the parameters involved in the neutrino masses, we consider the case where $\lambda_{2}, \lambda_{4}, \lambda_{7} \ll 1$ and $m_{\chi}^{2} \equiv m_{\eta^{ \pm}}^{2}=\mu_{\sigma}^{2} \gg \frac{1}{2} \lambda_{8} v v_{S}$, which leads to $a \approx b \gg c$. Taking into account that for the mentioned case $m_{\chi_{R_{2}}}^{2}-m_{\chi_{R_{1}}}^{2}=\lambda_{8} v v_{S}$ and $m_{\chi_{R_{2}}}^{2}+m_{\chi_{R_{1}}}^{2}=2 m_{\chi}^{2}$, we have that

$\left(\mathcal{M}_{\nu}\right)_{i j}=\frac{\lambda_{8} v_{S} v}{16 \pi^{2}} \sum_{\alpha=1}^{3} \frac{h_{i \alpha} M_{\psi_{\alpha}} y_{j \alpha}^{*}}{m_{\chi}^{2}-M_{\psi_{\alpha}}^{2}}\left[1-\frac{M_{\psi_{\alpha}}^{2}}{m_{\chi}^{2}-M_{\psi_{\alpha}}^{2}} \log \left(\frac{m_{\chi}^{2}}{M_{\psi_{\alpha}}^{2}}\right)\right]$,

and by further assuming $m_{\chi}^{2} \gg M_{\psi_{\alpha}}^{2}$, one finds

$$
\begin{gathered}
\left(\mathcal{M}_{\nu}\right)_{i j}=\frac{\lambda_{8} v_{S} v}{16 \pi^{2} m_{\chi}^{2}} \sum_{\alpha=1}^{3} h_{i \alpha} M_{\psi_{\alpha}} y_{j \alpha}^{*}, \\
\sim 0.04 \mathrm{eV}\left(\frac{\lambda_{8}}{10^{-4}}\right)\left(\frac{v_{S}}{200 \mathrm{GeV}}\right)\left(\frac{M_{\psi_{\alpha}}}{50 \mathrm{GeV}}\right) \\
\times\left(\frac{2 \mathrm{TeV}}{m_{\chi}}\right)^{2}\left(\frac{h_{i \alpha} y_{j \alpha}}{10^{-4}}\right) .
\end{gathered}
$$

In this way, in addition to the loop suppression, it is possible to have further suppression in the neutrino mass matrix for small values of either $v_{S}, \lambda_{8}$, or $h_{i \alpha} y_{j \alpha}$.

On the other hand, the $h_{i \alpha}$ Yukawa interaction in Eq. (2) leads to charged lepton flavor violation (CLFV) processes induced at one-loop level and mediated by the charged scalars $\eta^{ \pm}$as the ones shown in Fig. 2 for the $\ell_{i} \rightarrow \ell_{j} \gamma$ type. By using the current experimental constraint on $\operatorname{Br}(\mu \rightarrow e \gamma)<5.7 \times 10^{-13}$ [33] and for the case 


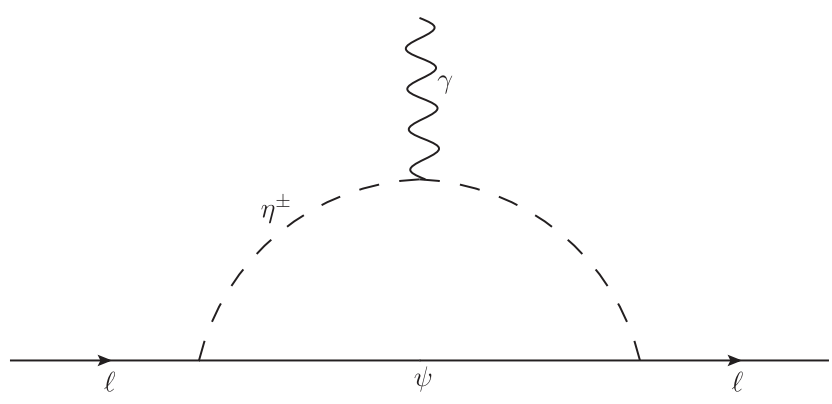

FIG. 2. Feynman diagram for the processes $\ell_{i} \rightarrow \ell_{j} \gamma$.

$m_{\chi}^{2}=m_{\eta^{ \pm}}^{2} \gg M_{\psi_{a}}^{2}$, we can obtain an upper bound for the product of Yukawa couplings,

$$
\left|\sum_{\alpha} h_{2 \alpha} h_{1 \alpha}^{*}\right| \lesssim 0.02\left(\frac{m_{\chi}}{2 \mathrm{TeV}}\right)^{2}
$$

It is worth noticing that $y_{j \alpha}$ is not constrained by the nonobservation of CLFV processes.

\section{DARK MATTER}

From the model charge assignment in Table I, we have that a residual $Z_{2}$ symmetry is left over after the $\mathrm{U}(1)_{\mathcal{D}}$ symmetry breaking, with the particles circulating the oneloop neutrino mass diagram (see Fig. 1) and $\nu_{R 3}$ being odd under it, whereas $\nu_{R 1}, \nu_{R 2}, S$, and all the SM particles being even. Thus, the lightest electrically neutral $Z_{2}$-odd particle becomes a dark matter candidate. In other words, this model also provides a solution to the DM puzzle via either fermion $\left(\psi_{1}\right)$ or scalar $\left(\chi_{1}\right)$ dark matter. ${ }^{2}$

Since $\psi_{1}$ is a singlet under the SM gauge group, its thermal relic density is controlled by the Yukawa couplings and $\mathrm{U}(1)_{\mathcal{D}}$ gauge interactions. The scenario where $\psi_{1}$ selfannihilates dominantly through $h_{i \alpha}$-mediated interactions resembles the very well-known scotogenic model [18], where sizable $h_{i \alpha}$ are required to reproduce the correct DM abundance, which in turn leads to a mild tension with the experimental upper bounds on the rates for rare charged lepton decays [34-36]. On the other hand, when the $Z^{\prime}$ portal $[37,38]$ is the main gate to the visible sector, $\psi_{1}$ largely annihilates into neutrinos in such a way that the observed DM abundance can be reproduced without entering into a conflict with the DM searches, which follows from the fact that the $Z^{\prime}$ does not couple to quarks and charged leptons (see [38-42] for phenomenological studies on $Z^{\prime}$-mediated Majorana DM).

\footnotetext{
${ }^{2}$ Note that $Z^{\prime}$ cannot constitute a DM candidate due to the instability associated to the $Z^{\prime} \rightarrow \bar{\nu}_{R 3} \nu_{R 3}$ decay channel, which cannot be kinematically closed, and that neither $\psi_{1}$ and $\chi_{1}$ can decay into $\nu_{R 3}$ since they do not share Yukawa interactions [see Eq. (2)].
}

The elastic scattering of $\psi_{1}$ particles off nuclei may occur via different mediators: inert scalars $(\sigma, \eta)$, SM Higgs (through the mixing with $S$ ), and $Z^{\prime}$. When the inert scalars mediate DM nucleon scatterings, the corresponding spin independent cross section is loop suppressed due to their quark-phobic nature [36], whereas the scatterings via the $Z^{\prime}$ require necessarily a kinetic mixing (since no SM fermion couples directly to $Z^{\prime}$ ) and lead to a spin-dependent cross section. Lastly, the scatterings via the Higgs exchange are also suppressed by the mixing parameter $\lambda_{1}$, which is required to be small to correctly reproduce the SM Higgs phenomenology. All in all, it is feasible to expect that the rates for the elastic scattering of $\psi$ particles off nuclei lie well below the sensitivity of present direct detection experiments.

In contrast to fermion DM, the scalar DM candidate has additional interaction terms to the Yukawa and gauge interactions. This entails that the later ones can be used to alter the relic density predictions in scenarios with mixed scalar DM. Since in the present model the $C P$-even and $C P$-odd neutral $Z_{2}$-odd particles are mass degenerate, we have the scenario of singlet-doublet complex DM [43,44], where the DM candidate is a mixture of a complex singlet [45] and a $S U(2)_{L}$ doublet [46,47]. It follows that for negligible Yukawa and gauge interactions, there are two DM mass regions that allow us to properly reproduce the relic abundance, one corresponds to the Higgs funnel region and the second one demands masses above $100 \mathrm{GeV}$ [48]. Regarding direct detection signals, we expect similar scattering rates as those in the complex scalar singlet model $[49,50]$.

\section{BEYOND THE MINIMAL MODEL: COSMOLOGICAL AND COLLIDER CONSTRAINTS}

It turns out that the $Z^{\prime}$ portal also allows us to probe the model through modifications to the cosmological history of the Universe, namely, via additional contributions to the effective number of relativistic d.o.f. $N_{\text {eff }}$ [51]. In this model, these contributions arise from the presence of the right-handed neutrinos and may be expected to be sizeable precisely due to the large $\mathrm{U}(1)_{\mathcal{D}}$ charges of the $\nu_{R}$ 's. Nevertheless, since the right-handed neutrinos do not couple directly to the rest of the SM particles [see Eq. (2)], they decouple early enough (when the DM does or even before) from the thermal bath and, therefore, modify the SM prediction for $N_{\text {eff }}$ with an extra contribution of at most $\sim 0.2$ [52].

With the aim of extending the thermalization period of the right-handed neutrinos with the primordial plasma in the early Universe, we consider an modification of the previous setup by using a general anomaly free Abelian gauge symmetry with generation-independent charge assignments for the SM fermions, $\mathrm{U}(1)_{X}$. In the Appendix, we show that the solutions to the anomaly 
TABLE II. General one-parameter solution with some examples of rational solutions $(X=B-L, R, D, G$, and $\mathcal{D})$ for the radiative type-I seesaw realization of the effective operator $\mathcal{O}_{6 D}$ for Dirac neutrino masses. The last column corresponds to the solution in Table I.

\begin{tabular}{lccccccc}
\hline \hline Fields & $S U(2)_{L}$ & $\mathrm{U}(1)_{Y}$ & $\mathrm{U}(1)_{X}$ & $\mathrm{U}(1)_{B-L}$ & $\mathrm{U}(1)_{R}$ & $\mathrm{U}(1)_{D}$ & $\mathrm{U}(1)_{G}$ \\
\hline$L$ & $\mathbf{2}$ & -1 & $l$ & -1 & 0 & $-3 / 2$ & $-1 / 2$ \\
$d_{R}$ & $\mathbf{1}$ & $-2 / 3$ & $1+2 l / 3$ & $1 / 3$ & 1 & 0 & $2 / 3$ \\
$u_{R}$ & $\mathbf{1}$ & $+4 / 3$ & $-1-4 l / 3$ & $1 / 3$ & -1 & 1 & $-1 / 3$ \\
$Q$ & $\mathbf{2}$ & $1 / 3$ & $-l / 3$ & $1 / 3$ & 0 & $1 / 2$ & $1 / 6$ \\
$e_{R}$ & $\mathbf{1}$ & -2 & $1+2 l$ & -1 & 1 & -2 & 0 \\
$H$ & $\mathbf{2}$ & 1 & $-1-l$ & 0 & -1 & $1 / 2$ & $-1 / 2$ \\
$\eta$ & $\mathbf{2}$ & 1 & $3 / 4-l$ & $7 / 4$ & $3 / 4$ & $9 / 4$ & $5 / 4$ \\
$S$ & $\mathbf{1}$ & 0 & $3 / 2$ & $3 / 2$ & $3 / 2$ & $3 / 2$ & $3 / 2$ \\
$\sigma$ & $\mathbf{1}$ & 0 & $13 / 4$ & $13 / 4$ & $13 / 4$ & $13 / 4$ & $13 / 4$ \\
$\nu_{R i}$ & $\mathbf{1}$ & 0 & -4 & -4 & -4 & -4 & -4 \\
$\nu_{R 3}$ & $\mathbf{1}$ & 0 & +5 & +5 & 5 & -4 & -4 \\
$\psi_{R \alpha}$ & $\mathbf{1}$ & 0 & $3 / 4$ & $3 / 4$ & $3 / 4$ & $3 / 4$ & 5 \\
$\xi_{L \alpha}$ & $\mathbf{1}$ & 0 & $3 / 4$ & $3 / 4$ & $3 / 4$ & $3 / 4$ & $3 / 4$ \\
\hline \hline
\end{tabular}

cancellation conditions allow us to write the $X$ charges of the SM fields in terms of two parameters [28,53-55] that we choose as

$$
X(r, l)=r R-l Y
$$

where $R$ is the generator of $\mathrm{U}(1)_{R}$ (the gauge Abelian symmetry where only the right-handed SM fermions have nonvanishing $X$ charges), $Y$ is the hypercharge, $l$ is the $X$ charge of the lepton doublets, and $r$ parametrizes the contribution to the linear and mixed gauge-gravitational anomalies of any extra set of chiral fermions, as given in Eq. (A4) of the Appendix. In this way, if only extra vectorlike fermions are allowed beyond the SM $(r=0)$, the solution must be proportional to the hypercharge. Without a loss of generality, we can write the solutions in terms of just one parameter [56-58] that we choose to be $l$ after fix $r=1$. Then, as shown in the Appendix, the full family of solutions for a fixed $l$ can be obtained after rescaling all the $X$ charges by a factor $r$. In particular, the fermiophobic [28-30] solution used in the previous sections, $\mathrm{U}(1)_{\mathcal{D}}$, corresponds to the rescaling $r=0$ of the $\mathrm{U}(1)_{R}$ solution: $\mathcal{D}=X(0,0)$.

The one parameter solution is shown in column $\mathrm{U}(1)_{X}$ of Table II. In order to analyze the phenomenology, we fix $l$ to recover some already studied Abelian gauge groups $X=B-L, R, D, G$, as defined in Ref. [28]. ${ }^{3}$ The last column corresponds to the rescaling with $r=0$ of $\mathrm{U}(1)_{R}$.

Since we are interested here in keeping the exotic set of $X$-charges charges $\{\mp 4, \mp 4, \pm 5\}$ in such a way that when assigned to the right-handed neutrinos, the tree level Dirac and Majorana masses can be forbidden [24], the set of Majorana mediators $\psi_{R \alpha}$, which realize the dimension-6 operator at one-loop, would spoil the anomaly cancellation condition. In view of that $\psi_{R \alpha}$ have necessarily nonzero $X$

\footnotetext{
${ }^{3}$ We change $\mathrm{U}(1)_{B}$ for the more suitable name of $\mathrm{U}(1)_{R}[59]$.
}

charges (see the Appendix), we further add an extra set of chiral fermions in such a way the full set heavy fermions do not affect the anomaly cancellation (their charges cancel each other in a vectorlike way). The resulting charge assignment is displayed in Table II, where the fields $\xi_{L \alpha}$ constitute the new set of chiral fermion that guarantee the anomaly cancellation. In this way, we end up with a model with four Majorana fermions, two more than in the minimal solution, since the third generation of chiral fermions is not needed to cancel the $\mathrm{U}(1)_{X}$ anomalies (alternatively, we may consider another simple setup by adding a single set of $\psi_{R}$ and $\xi_{L}$ and two set of scalars $\left.\eta_{\alpha}, \sigma_{\alpha}[60]\right){ }^{4}$

Accordingly, we may expect that the three right-handed neutrinos within the not-so minimal model contribute to the radiation energy density of the Universe, since now the interaction between the $Z^{\prime}$ with the SM fermions opens up the possibility to thermalize them with the primordial plasma. In other words, this leads to a modification in the relativistic d.o.f. as $[61,62]$

$$
\Delta N_{\mathrm{eff}}=N_{\mathrm{eff}}-N_{\mathrm{eff}}^{\mathrm{SM}}=N_{\nu_{R}}\left(\frac{T_{\nu_{R}}}{T_{\nu_{L}}}\right)^{4}=N_{\nu_{R}}\left(\frac{g\left(T_{\mathrm{dec}}^{\nu_{L}}\right)}{g\left(T_{\mathrm{dec}}^{\nu_{R}}\right)}\right)^{4 / 3}
$$

where $N_{\nu_{R}}$ is the number of right-handed neutrinos with the same $X$ charge and $g(T)$ is the number of relativistic d.o.f. at a temperature $T$ in the SM [63]. The decoupling temperature of the SM neutrinos is $T_{\mathrm{dec}}^{\nu_{L}} \approx 2.3 \mathrm{MeV}$, when $g\left(T_{\mathrm{dec}}^{\nu_{L}}\right)=43 / 4$ corresponding to the three $\nu_{L}, e^{ \pm}$and the

\footnotetext{
${ }^{4}$ Seeing that the two additional left-handed fields $\xi_{L \alpha}$, with an $X$ charge $r=3 / 4$, have both Dirac and Majorana mass terms $\overline{\xi_{L \alpha}} \psi_{R \beta}$ and $\overline{\xi_{L \alpha}^{c}} \xi_{L \beta}\langle S\rangle$, the full set of Majorana fields are massive and heavy. Moreover, because the $\mathrm{U}(1)_{X}$ left out a remnant $Z_{2}$ discrete symmetry which guarantees the stability of the lightest $Z_{2}$-odd particle, we have that the lightest of these Majorana fields may play the role of a DM candidate.
} 
photon $[64,65]$. Since the interaction of the right-handed neutrinos with the SM is only mediated by the gauge boson $Z^{\prime}$, the corresponding rate can be cast as [66]

$$
\begin{aligned}
\Gamma_{\nu_{R}}(T) & =n_{\nu_{R}}(T)\left\langle\sigma\left(\overline{\nu_{R}} \nu_{R} \rightarrow \bar{f} f\right) v\right\rangle \\
& =\frac{g_{\nu_{R}}^{2}}{n_{\nu_{R}}(T)} \int \frac{d^{3} p}{(2 \pi)^{3}} f_{\nu_{R}}(p) \int \frac{d^{3} k}{(2 \pi)^{3}} f_{\nu_{R}}(k) \sigma_{f}(s) v,
\end{aligned}
$$

where $f_{\nu_{R}}(k)=1 /\left(e^{k / T}+1\right)$ is the Fermi-Dirac distribution, $g_{\nu_{R}}=2, \quad v=1-\cos \varphi$ is the Moller velocity, $s=$ $2 p k(1-\cos \varphi), p$ and $k$ are the momenta of the particle with $\varphi$ the angle between them, and the number density of right-handed neutrinos is given by

$$
n_{\nu_{R}}(T)=g_{\nu_{R}} \int \frac{d^{3} k}{(2 \pi)^{3}} f_{\nu_{R}}(k)
$$

The cross section for the case of a heavy mediator $\left(T_{\mathrm{dec}}^{\nu_{R}} \ll\right.$ $M_{Z^{\prime}}$ ) with $s \ll M_{Z^{\prime}}$ and neglecting the fermion masses in the final state reads [67]

$$
\sigma_{f}(s) \approx N_{f}^{C} \frac{s}{12 \pi}\left(\frac{g^{\prime}}{M_{Z^{\prime}}}\right)^{4} q_{\nu_{R}}^{2}\left(q_{f_{L}}^{2}+q_{f_{R}}^{2}\right),
$$

where $N_{f}^{C}=1(3)$ for leptons (quarks), and $q_{f}$ is the $X$ charge of the SM fermion. Accordingly, the interaction rate takes the form,

$$
\Gamma_{\nu_{R}}(T)=\frac{49 \pi^{5} T^{5}}{97200 \zeta(3)}\left(\frac{g^{\prime}}{M_{Z^{\prime}}}\right)^{4} \sum_{f} N_{f}^{C} q_{f}^{2}
$$

In this expression, the sum is performed over all SM fermions that are in thermal equilibrium with the plasma at temperature $T$. To estimate the contribution to the relativistic d.o.f. from right-handed neutrinos, it is necessary to calculate the decoupling temperature of the right-handed neutrinos $\left(T_{\mathrm{dec}}^{\nu_{R}}\right)$. The latter occurs when the interaction rate $\Gamma_{\nu_{R}}(T)$ drops below the rate of expansion of the Universe, $\Gamma\left(T_{\mathrm{dec}}^{\nu_{R}}\right)=H\left(T_{\mathrm{dec}}^{\nu_{R}}\right)$, with $H(T)=\left[4 \pi^{3} G_{N}(g(T)+21 / 4) / 45\right]^{1 / 2} T^{2}$. Note that the factor $21 / 4$ corresponds to the contribution of the righthanded neutrinos to the relativistic d.o.f.

The results for $\Delta N_{\text {eff }}$ as a function of $M_{Z^{\prime}} / g^{\prime}$ are displayed in Fig. 3, where it can be observed that for the small ratios $M_{Z^{\prime}} / g^{\prime}$ the variation in the number of relativistic d.o.f. of the new species is large $\left(\Delta N_{\text {eff }} \gtrsim 0.3\right.$ corresponds to decoupling temperatures $T_{\text {dec }}^{\nu_{R}} \lesssim 2 \mathrm{GeV}$ ) and vice versa. It follows that for the $\mathrm{U}(1)_{G}$ model, the Planck + BAO upper bound at $2 \sigma$ (solid gray line) demands that $M_{Z^{\prime}} / g^{\prime} \gtrsim 36 \mathrm{TeV}$, while for the $\mathrm{U}(1)_{D}$ model, a more stringent lower bound applies $M_{Z^{\prime}} / g^{\prime} \gtrsim$ $55 \mathrm{TeV}$ (these bounds become slightly weaker once the

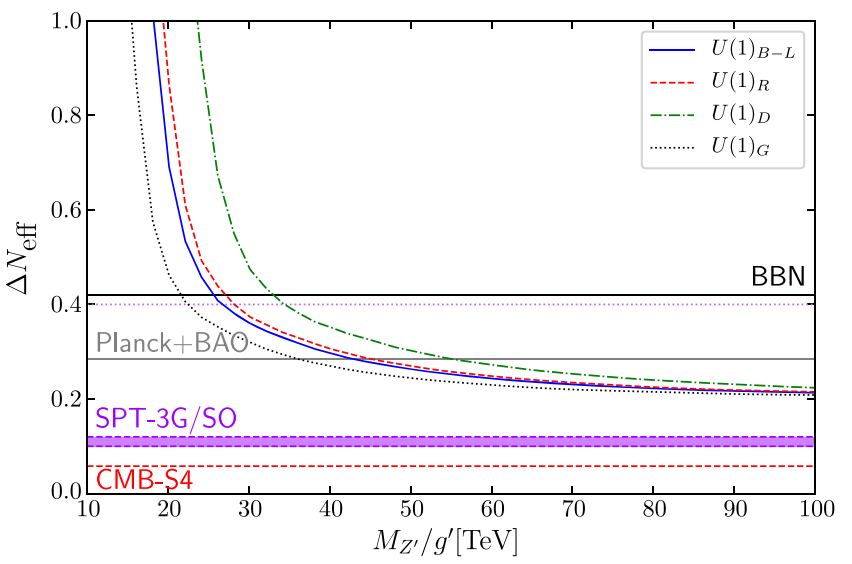

FIG. 3. Contribution to the number of extra relativistic d.o.f. $\left(\Delta N_{\text {eff }}\right)$ in the function of $M_{Z^{\prime}} / g^{\prime}$. The region above the solid gray line is excluded by the measurements at $2 \sigma$ reported by the PLANCK Collaboration [68]. For comparison purposes, the upper bound at $2 \sigma$ (black line) obtained from the BBN analysis [69] is also shown. The horizontal dashed lines show the projected sensitivity of the future experiments SPT-3G/SO $[74,75]$ and CMB-S4 [76]. $\Delta N_{\text {eff }}=0.4$ represents the extra contribution required to relieve the tension on the inferred $H_{0}$ values from high- and low-redshift observations $[79,80]$.

Planck $+\mathrm{BAO}+H_{0}$ combination [68] is considered, but in such a case, the BBN bound from the primordial abundances of light elements would rule [69]). In other words, bearing in mind that $M_{Z^{\prime}} / g^{\prime} \approx 3 v_{S} / 2,{ }^{5}$ we have that the energy scale of the $\mathrm{U}(1)_{X}$ symmetry breaking must be at least $\sim 24 \mathrm{TeV}$ [note that the $\mathrm{U}(1)_{X}$ having the lowest $M_{Z^{\prime}} / g^{\prime}$ ratio features a $\mathrm{X}$ charge $\left.l=-6 / 11 \approx-1 / 2\right]$. Similar constraints would apply to all gauged and anomaly free $\mathrm{U}(1)_{X}$ extensions of the SM with Dirac neutrino masses, where light right-handed neutrinos are associated to the solution $(-4,-4,+5)[17,24,25,71-73]$. On the other hand, it is remarkable the fact the next generation of CMB experiments [74-76] has the potential to entirely probe all the not-so minimal models $[77,78]$.

Regarding collider searches, the recasting of the latest ATLAS results for the search of dilepton resonances using $139 \mathrm{fb}^{-1}$ [81] was done in Ref. [82] for the $\mathrm{U}(1)_{B-L}$ model. The green (upper) region in Fig. 4 shows the excluded region at $95 \%$ C.L. To ease the comparison with other results, we show the exclusion as function of $M_{Z^{\prime}} / g^{\prime}$. In particular, the limit from LEP for $\mathrm{U}(1)_{B-L}$ model is $[70,83,84]^{6}$

$$
M_{Z^{\prime}} / g^{\prime}>6.7 \mathrm{TeV}, \quad \text { at } 95 \% \text { C.L. }
$$

which is obtained from the search for effective four-lepton operators and is valid for $M_{Z^{\prime}} \gg 200 \mathrm{GeV}$. This constraint

\footnotetext{
${ }^{5}$ Because of the LEP constraint (see below), the mixing between the $Z^{\prime}$ and the SM $Z$ boson is negligible [70].

${ }^{6}$ The constraint as a function of the $X$ charge $h=-1-l$ is given in Ref. [85].
} 


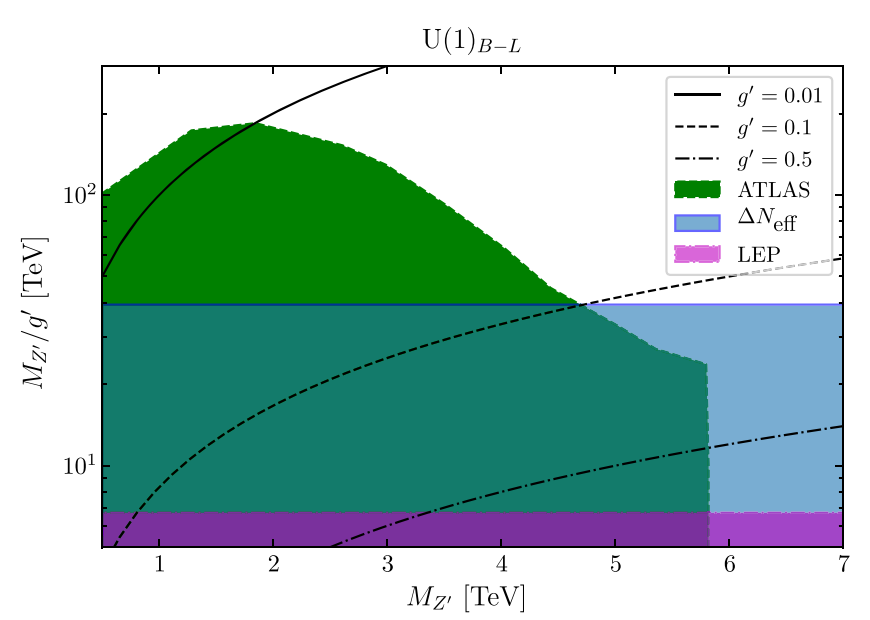

FIG. 4. Collider and cosmological constraints for the $\mathrm{U}(1)_{B-L}$ model, and contours of constant $g^{\prime}$ in the plane of $q_{S} v_{S}$ as a function of $M_{Z^{\prime}}$ with $q_{S}=3 / 2$.

corresponds to the excluded magenta (lower) region of Fig. 4 and start to be relevant for $M_{Z^{\prime}}>5.8 \mathrm{TeV}$.

The constraint of $\Delta N_{\text {eff }}$ for $\mathrm{U}(1)_{B-L}$ is shown in the blue (middle) region and start to be better than the current ATLAS limit for $M_{Z^{\prime}} \gtrsim 4.8 \mathrm{TeV}$. In the figure, we also show contours of constant $g^{\prime}$ at $0.01,0.1$, and 0.5 with the solid, dashed, and dot-dashed lines respectively. We can see that $\Delta N_{\text {eff }}$ start to constrain $g^{\prime}$ for values larger than 0.1 in $\mathrm{U}(1)_{B-L}$. Similar restrictions can be obtained for the other models quoted in Fig. 3.

\section{CONCLUSIONS}

The mechanism behind the neutrino mass generation and the Dirac/Majorana character of massive neutrinos still remain a conundrum despite the clear understanding of the neutrino oscillation pattern and the great experimental efforts behind the neutrinoless double-beta decay. Alongside this is the fact that there are no clues on the nature of the DM particle and the properties of the dark sector it belongs.

In view of this, we have proposed a new mechanism for Dirac neutrino masses which makes use of heavy Majorana mediators that generate nonzero masses at one-loop level. The model presented in this paper is a one-loop realization of the dimension 6 operator $\bar{L} \tilde{H} \nu_{R} S^{2}$ and enters into the list of Dirac radiative type-I seesaw models with the novel feature that it involves Majorana mediators rather than Dirac mediators. The Dirac-ness of the massive neutrinos is protected by only one extra $\mathrm{U}(1)_{X}$ gauge symmetry, which in turn ensures the stability of the lightest particle mediating the one-loop neutrino mass diagram. In this way, the model offers, in a nontrivial way, the solution to both neutrino and DM puzzles. Moreover, we have shown that going beyond the minimal model other interesting phenomenological aspects arise, such as possible signals at colliders and new contributions to the number of extra relativistic species. In particular, the current upper bound on $\Delta N_{\text {eff }}$ reported by PLANCK points to large ratios $M_{Z^{\prime}} / g^{\prime} \gtrsim$ $40 \mathrm{TeV}$. Future cosmic microwave background experiments may probe all the no minimal models presented here.

The supporting data are available in Ref. [86].

\section{ACKNOWLEDGMENTS}

Work supported by Sostenibilidad-UdeA and the UdeA/ CODI Grant No. 2017-16286, and by COLCIENCIAS through the Grant No. 111577657253. D. R. would also like to thank the IIP-UFRN, for its support, hospitality, and the nice and stimulating atmosphere during the Visiting Professors program where part of this work was completed. O.Z. acknowledges the ICTP Simons associates program.

\section{APPENDIX: U(1) $)_{X}$ ANOMALY CANCELLATION}

We use $f(f)$ to denote the general $\mathrm{U}(1)_{X}$ generationindependent charge assignments of the field $f_{R}\left(F_{L}\right)$. The three linear anomalies in $\mathrm{U}(1)_{X}[28]$,

$$
\begin{aligned}
& {\left[\mathrm{SU}(3)_{C}\right]^{2} \mathrm{U}(1)_{X}: } {[3 u+3 d]-[3 \cdot 2 q]=0, } \\
& {\left[\mathrm{SU}(2)_{L}\right]^{2} \mathrm{U}(1)_{X}:-[2 l+3 \cdot 2 q]=0, } \\
& {\left[\mathrm{U}(1)_{Y}\right]^{2} \mathrm{U}(1)_{X}:\left[(-2)^{2} e+3\left(\frac{4}{3}\right)^{2} u+3\left(-\frac{2}{3}\right)^{2} d\right] } \\
&-\left[2(-1)^{2} l+3 \cdot 2\left(\frac{1}{3}\right)^{2} q\right]=0,
\end{aligned}
$$

allows us to express three $X$ charges in terms of the other two,

$$
u=-e+\frac{2 l}{3}, \quad d=e-\frac{4 l}{3}, \quad q=-\frac{l}{3} .
$$

The quadratic anomaly condition is automatically satisfied, while the mixed gauge-gravitational and cubic anomalies depend on any extra singlet quiral fermions of zero hypercharge, like the right-handed counterpart of the Dirac neutrinos. For $N$ extra quiral fields with an $X$ charge $n_{\alpha}$, these conditions read

$$
\begin{array}{r}
{[\mathrm{Grav}]^{2} \mathrm{U}(1)_{X}: \sum_{\alpha=1}^{N} n_{\alpha}+3(e-2 l)=0,} \\
{\left[\mathrm{U}(1)_{X}\right]^{3}: \sum_{\alpha=1}^{N} n_{\alpha}^{3}+3(e-2 l)^{3}=0 .}
\end{array}
$$

We choose the solutions with $r \equiv e-2 l$, such that

$$
\sum_{\alpha=1}^{N} n_{\alpha}=-3 r, \quad \sum_{\alpha=1}^{N} n_{\alpha}^{3}=-3 r^{3} .
$$

The full set of anomaly free SM $X$ charges in terms of two parameters $[28,53,54]$ that we choose as $l$ and $r$, is just 
$u=-r-\frac{4 l}{3}, \quad d=r+\frac{2 l}{3}, \quad q=-\frac{l}{3}$,

$e=r+2 l, \quad h=-r-l$,

where the condition in the charged lepton Yukawa couplings have been used to fix $h$ and is automatically consistent with the conditions in the quark Yukawa couplings. By setting $l=0$ in the previous equations, we can define the Abelian symmetry in which only the right-handed charged fermions have nonvanishing $X$ charges as $\mathrm{U}(1)_{R}$. Then the general anomaly free twoparameter solution can be written as

$$
X(r, l)=r R-l Y .
$$

If we now change $f \rightarrow f^{\prime}=f / r$ for all the charged fermion $X$ charges [54], the first set of anomaly cancellation conditions Eq. (A1) remains invariant, and without a loss of generality, it is always possible to normalize the solutions such that the last set Eq. (A4) is just

$$
\sum_{\alpha=1}^{N} n_{\alpha}^{\prime}=-3, \quad \sum_{\alpha=1}^{N} n_{\alpha}^{\prime 3}=-3 .
$$

[1] M. Tanabashi et al. (Particle Data Group), Review of particle physics, Phys. Rev. D 98, 030001 (2018).

[2] A. Gando et al. (KamLAND-Zen Collaboration), Search for Majorana Neutrinos near the Inverted Mass Hierarchy Region with KamLAND-Zen, Phys. Rev. Lett. 117, 082503 (2016); 117, 109903(A) (2016).

[3] M. Agostini et al. (GERDA Collaboration), Improved Limit on Neutrinoless Double- $\beta$ Decay of ${ }^{76} \mathrm{Ge}$ from GERDA Phase II, Phys. Rev. Lett. 120, 132503 (2018).

[4] C. E. Aalseth et al. (Majorana Collaboration), Search for Neutrinoless Double- $\beta$ Decay in ${ }^{76} \mathrm{Ge}$ with the Majorana Demonstrator, Phys. Rev. Lett. 120, 132502 (2018).

[5] C. Alduino et al. (CUORE Collaboration), First Results from CUORE: A Search for Lepton Number Violation via $0 \nu \beta \beta$ Decay of ${ }^{130} \mathrm{Te}$, Phys. Rev. Lett. 120, 132501 (2018).

[6] J. B. Albert et al. (EXO Collaboration), Search for Neutrinoless Double-Beta Decay with the Upgraded EXO-200 Detector, Phys. Rev. Lett. 120, 072701 (2018).

[7] R. Arnold et al. (NEMO-3 Collaboration), Measurement of the $2 \nu \beta \beta$ decay half-life and search for the $0 \nu \beta \beta$ decay of ${ }^{116} \mathrm{Cd}$ with the NEMO-3 detector, Phys. Rev. D 95, 012007 (2017).

[8] Q. R. Ahmad et al. (SNO Collaboration), Direct Evidence for Neutrino Flavor Transformation from Neutral Current Interactions in the Sudbury Neutrino Observatory, Phys. Rev. Lett. 89, 011301 (2002).
For example, the solution with $r=3: n_{\alpha}=(-2,-2,-4,-1)$ [53] can be easily normalized to the form in Eq. (A7) with $f \rightarrow f / 3$ to $n_{\alpha}^{\prime}=(-2 / 3,-2 / 3,-4 / 3,-1 / 3)$ as used in Ref. [87]. In this way, without the loss of generality, we will work with the normalized solution in terms of a single parameter [56-58] that we choose to be $l$, by setting $r=1$ as summarized in column $\mathrm{U}(1)_{X}$ of Table II, which is just

$$
X(l)=R-l Y .
$$

In particular, this includes the solution $n_{\alpha}=(-4,-4,+5)$ [53]. In general, we have that for $\nu=n_{1}=n_{2}$, the extra fermion inside the one-loop neutrino mass diagram in Fig. 1, must have the charges,

$$
\psi=-\frac{\nu+1}{4}, \quad \eta=-\frac{\nu+1}{4}-l, \quad \sigma=\frac{1-3 \nu}{4} .
$$

The case for $\nu=-4$ is also displayed in Table II.
[9] Y. Fukuda et al. (Super-Kamiokande Collaboration), Evidence for Oscillation of Atmospheric Neutrinos, Phys. Rev. Lett. 81, 1562 (1998).

[10] P. Minkowski, $\mu \rightarrow e \gamma$ at a rate of one out of $10^{9}$ muon decays? Phys. Lett. 67B, 421 (1977).

[11] T. Yanagida, Horizontal gauge symmetry and masses of neutrinos, Conf. Proc. C 7902131, 95 (1979).

[12] M. Gell-Mann, P. Ramond, and R. Slansky, Complex spinors and unified theories, Conf. Proc. C 790927, 315 (1979).

[13] R. N. Mohapatra and G. Senjanovic, Neutrino Mass and Spontaneous Parity Nonconservation, Phys. Rev. Lett. 44, 912 (1980).

[14] M. Roncadelli and D. Wyler, Naturally Light Dirac Neutrinos in Gauge Theories, Phys. Lett. 133B, 325 (1983).

[15] P. Roy and O. U. Shanker, Observable Neutrino Dirac Mass and Supergrand Unification, Phys. Rev. Lett. 52, 713 (1984); Erratum, Phys. Rev. Lett. 52, 2190(E) (1984).

[16] P.-H. Gu, H.-J. He, and U. Sarkar, Realistic neutrinogenesis with radiative vertex correction, Phys. Lett. B 659, 634 (2008).

[17] E. Ma and R. Srivastava, Dirac or inverse seesaw neutrino masses with $B-L$ gauge symmetry and $S_{3}$ flavor symmetry, Phys. Lett. B 741, 217 (2015).

[18] E. Ma, Verifiable radiative seesaw mechanism of neutrino mass and dark matter, Phys. Rev. D 73, 077301 (2006). 
[19] Y. Farzan and E. Ma, Dirac neutrino mass generation from dark matter, Phys. Rev. D 86, 033007 (2012).

[20] E. Ma, I. Picek, and B. Radovčić, New scotogenic model of neutrino mass with $\mathrm{U}(1)_{D}$ gauge interaction, Phys. Lett. B 726, 744 (2013),

[21] D. A. Demir, L. L. Everett, and P. Langacker, Dirac Neutrino Masses from Generalized Supersymmetry Breaking, Phys. Rev. Lett. 100, 091804 (2008).

[22] Z.-L. Han and W. Wang, $Z^{\prime}$ portal dark matter in $B-L$ scotogenic dirac model, Eur. Phys. J. C 78, 839 (2018).

[23] W. Wang, R. Wang, Z.-L. Han, and J.-Z. Han, The $B-L$ scotogenic models for dirac neutrino masses, Eur. Phys. J. C 77, 889 (2017).

[24] J. Calle, D. Restrepo, C. E. Yaguna, and Ó. Zapata, Minimal radiative Dirac neutrino mass models, Phys. Rev. D 99, 075008 (2019).

[25] C. Bonilla, S. Centelles-Chuliá, R. Cepedello, E. Peinado, and R. Srivastava, Dark matter stability and Dirac neutrinos using only Standard Model symmetries, arXiv:1812.01599.

[26] S. Saad, Simplest radiative dirac neutrino mass models, Nucl. Phys. B943, 114636 (2019).

[27] G. Bertone, D. Hooper, and J. Silk, Particle dark matter: Evidence, candidates and constraints, Phys. Rep. 405, 279 (2005).

[28] M. D. Campos, D. Cogollo, M. Lindner, T. Melo, F. S. Queiroz, and W. Rodejohann, Neutrino masses and absence of flavor changing interactions in the $2 \mathrm{HDM}$ from gauge principles, J. High Energy Phys. 08 (2017) 092.

[29] E. Bertuzzo, S. Jana, P. A. N. Machado, and R. Zukanovich Funchal, Dark Neutrino Portal to Explain MiniBooNE excess, Phys. Rev. Lett. 121, 241801 (2018).

[30] E. Bertuzzo, S. Jana, P. A. N. Machado, and R. Zukanovich Funchal, Neutrino masses and mixings dynamically generated by a light dark sector, Phys. Lett. B 791, 210 (2019).

[31] S. Centelles Chuliá, E. Ma, R. Srivastava, and J. W. F. Valle, Dirac neutrinos and dark matter stability from lepton quarticity, Phys. Lett. B 767, 209 (2017).

[32] P. F. de Salas, D. V. Forero, C. A. Ternes, M. Tórtola, and J. W. F. Valle, Status of neutrino oscillations 2018: $3 \sigma$ hint for normal mass ordering and improved $C P$ sensitivity, Phys. Lett. B 782, 633 (2018).

[33] J. Adam et al. (MEG Collaboration), New Constraint on the Existence of the $\mu^{+} \rightarrow e^{+} \gamma$ Decay, Phys. Rev. Lett. 110, 201801 (2013).

[34] J. Kubo, E. Ma, and D. Suematsu, Cold dark matter, radiative neutrino mass, $\mu \rightarrow e \gamma$, and neutrinoless double beta decay, Phys. Lett. B 642, 18 (2006).

[35] D. Aristizabal Sierra, J. Kubo, D. Restrepo, D. Suematsu, and O. Zapata, Radiative seesaw: Warm dark matter, collider and lepton flavour violating signals, Phys. Rev. D 79, 013011 (2009).

[36] A. Ibarra, C. E. Yaguna, and O. Zapata, Direct detection of fermion dark matter in the radiative seesaw model, Phys. Rev. D 93, 035012 (2016).

[37] P. Langacker, The physics of heavy $Z^{\prime}$ gauge bosons, Rev. Mod. Phys. 81, 1199 (2009).

[38] G. Arcadi, Y. Mambrini, M. H. G. Tytgat, and B. Zaldivar, Invisible $Z^{\prime}$ and dark matter: LHC vs LUX constraints, J. High Energy Phys. 03 (2014) 134.
[39] A. Alves, S. Profumo, and F. S. Queiroz, The dark $Z^{\prime}$ portal: direct, indirect and collider searches, J. High Energy Phys. 04 (2014) 063.

[40] A. Alves, A. Berlin, S. Profumo, and F. S. Queiroz, Dark matter complementarity and the $Z^{\prime}$ portal, Phys. Rev. D 92, 083004 (2015).

[41] A. Alves, G. Arcadi, Y. Mambrini, S. Profumo, and F. S. Queiroz, Augury of darkness: The low-mass dark $Z^{\prime}$ portal, J. High Energy Phys. 04 (2017) 164.

[42] C. Blanco, M. Escudero, D. Hooper, and S. J. Witte, $Z^{\prime}$ mediated WIMPs: Dead, dying, or soon to be detected?, J. Cosmol. Astropart. Phys. 11 (2019) 024.

[43] M. Kadastik, K. Kannike, and M. Raidal, Matter parity as the origin of scalar Dark Matter, Phys. Rev. D 81, 015002 (2010).

[44] G. Belanger, K. Kannike, A. Pukhov, and M. Raidal, Impact of semi-annihilations on dark matter phenomenology-an example of $Z_{N}$ symmetric scalar dark matter, J. Cosmol. Astropart. Phys. 04 (2012) 010.

[45] J. McDonald, Gauge singlet scalars as cold dark matter, Phys. Rev. D 50, 3637 (1994).

[46] N. G. Deshpande and E. Ma, Pattern of symmetry breaking with two higgs doublets, Phys. Rev. D 18, 2574 (1978).

[47] R. Barbieri, L. J. Hall, and V.S. Rychkov, Improved naturalness with a heavy Higgs: An Alternative road to LHC physics, Phys. Rev. D 74, 015007 (2006).

[48] M. Kakizaki, A. Santa, and O. Seto, Phenomenological signatures of mixed complex scalar WIMP dark matter, Int. J. Mod. Phys. A 32, 1750038 (2017).

[49] J. M. Cline, K. Kainulainen, P. Scott, and C. Weniger, Update on scalar singlet dark matter, Phys. Rev. D 88, 055025 (2013); Erratum, Phys. Rev. D 92, 039906(E) (2015).

[50] H. Wu and S. Zheng, Scalar dark matter: Real vs complex, J. High Energy Phys. 03 (2017) 142.

[51] A. D. Dolgov, Neutrinos in cosmology, Phys. Rep. 370, 333 (2002).

[52] Z. Chacko, Y. Cui, S. Hong, and T. Okui, Hidden dark matter sector, dark radiation, and the CMB, Phys. Rev. D 92, 055033 (2015).

[53] T. Appelquist, B. A. Dobrescu, and A. R. Hopper, Nonexotic neutral gauge bosons, Phys. Rev. D 68, 035012 (2003).

[54] B. C. Allanach, J. Davighi, and S. Melville, An Anomalyfree Atlas: charting the space of flavour-dependent gauged $\mathrm{U}(1)$ extensions of the Standard Model, J. High Energy Phys. 02 (2019) 082; 08 (2019) 64.

[55] A. Das, S. Goswami, K. N. Vishnudath, and T. Nomura, Constraining a general $\mathrm{U}(1)^{\prime}$ inverse seesaw model from vacuum stability, dark matter and collider, arXiv:1905. 00201.

[56] E. E. Jenkins, Searching for a $\left(B^{-} 1\right)$ gauge boson in $p \bar{p}$ collisions, Phys. Lett. B 192, 219 (1987).

[57] S. Oda, N. Okada, and D.-S. Takahashi, Classically conformal U(1)' extended standard model and Higgs vacuum stability, Phys. Rev. D 92, 015026 (2015).

[58] N. Okada, S. Okada, and D. Raut, Natural Z' -portal Majorana dark matter in alternative U(1) extended standard model, Phys. Rev. D 100, 035022 (2019). 
[59] S. Jana, P. K. Vishnu, and S. Saad, Minimal dirac neutrino mass models from $\mathrm{U}(1)_{R}$ gauge symmetry and leftright asymmetry at collider, Eur. Phys. J. C 79, 916 (2019).

[60] M. Reig, D. Restrepo, J. W. F. Valle, and O. Zapata, Boundstate dark matter and Dirac neutrino masses, Phys. Rev. D 97, 115032 (2018).

[61] L. A. Anchordoqui, H. Goldberg, and G. Steigman, Righthanded neutrinos as the dark radiation: status and forecasts for the LHC, Phys. Lett. B 718, 1162 (2013).

[62] L. A. Anchordoqui and H. Goldberg, Neutrino Cosmology After WMAP 7-Year Data and LHC First Z' Bounds, Phys. Rev. Lett. 108, 081805 (2012).

[63] S. Borsanyi et al., Calculation of the axion mass based on high-temperature lattice quantum chromodynamics, Nature (London) 539, 69 (2016).

[64] E. W. Kolb and M.S. Turner, The early universe, Front. Phys. 69, 1 (1990).

[65] K. Enqvist, K. Kainulainen, and V. Semikoz, Neutrino annihilation in hot plasma, Nucl. Phys. B374, 392 (1992).

[66] A. Solaguren-Beascoa and M. C. Gonzalez-Garcia, Dark radiation confronting LHC in Z' models, Phys. Lett. B 719, 121 (2013).

[67] V. Barger, P. Langacker, and H.-S. Lee, Primordial nucleosynthesis constraints on $Z^{\prime}$ properties, Phys. Rev. D 67, 075009 (2003).

[68] N. Aghanim et al. (Planck Collaboration). Planck 2018 results. VI. Cosmological parameters, arXiv:1807.06209.

[69] C. Pitrou, A. Coc, J.-P. Uzan, and E. Vangioni, Precision big bang nucleosynthesis with improved Helium-4 predictions, Phys. Rep. 754, 1 (2018).

[70] M. Carena, A. Daleo, B. A. Dobrescu, and T. M. P. Tait, Z' gauge bosons at the Tevatron, Phys. Rev. D 70, 093009 (2004).

[71] E. Ma, N. Pollard, R. Srivastava, and M. Zakeri, Gauge $B-L$ model with residual $Z_{3}$ symmetry, Phys. Lett. B 750, 135 (2015).

[72] E. Ma and R. Srivastava, Dirac or inverse seesaw neutrino masses from gauged $B-L$ symmetry, Mod. Phys. Lett. A 30, 1530020 (2015).

[73] C. Bonilla, E. Peinado, and R. Srivastava, The role of residual symmetries in dark matter stability and the neutrino nature, Letters in High Energy Physics 124, 1 (2019).

[74] B. A. Benson et al. (SPT-3G Collaboration), SPT-3G: a next-generation cosmic microwave background polarization experiment on the south pole telescope, SPIE Int. Soc. Opt. Eng. 9153, 91531P (2014).

[75] J. Aguirre et al. (Simons Observatory Collaboration). The simons observatory: Science goals and forecasts, J. Cosmol. Astropart. Phys. 02 (2019) 056.

[76] M. H. Abitbol et al. (Simons Observatory Collaboration), The simons observatory: Astro2020 decadal project whitepaper, Bull. Am. Astron. Soc. 51, 147 (2019).

[77] D. Baumann, D. Green, and B. Wallisch, Searching for light relics with large-scale structure, J. Cosmol. Astropart. Phys. 08 (2018) 029.

[78] K. N. Abazajian and J. Heeck, Observing Dirac neutrinos in the cosmic microwave background, Phys. Rev. D 100, 075027 (2019).

[79] J. L. Bernal, L. Verde, and A. G. Riess, The trouble with $H_{0}$, J. Cosmol. Astropart. Phys. 10 (2016) 019.

[80] E. Mörtsell and S. Dhawan, Does the Hubble constant tension call for new physics?. J. Cosmol. Astropart. Phys. 09 (2018) 025.

[81] G. Aad et al. (ATLAS Collaboration), Search for high-mass dilepton resonances using $139 \mathrm{fb}^{-1}$ of $p p$ collision data collected at $\sqrt{s}=13 \mathrm{TeV}$ with the ATLAS detector, Phys. Lett. B 796, 68 (2019).

[82] C.-W. Chiang, G. Cottin, A. Das, and S. Mandal, Displaced heavy neutrinos from $Z^{\prime}$ decays at the LHC, J. High Energy Phys. 12 (2019) 070.

[83] J. Heeck, Unbroken B-L symmetry, Phys. Lett. B 739, 256 (2014).

[84] A. Das, N. Okada, and N. Papapietro, Electroweak vacuum stability in classically conformal B-L extension of the Standard Model, Eur. Phys. J. C 77, 122 (2017).

[85] N. Okada and S. Okada, $Z^{\prime}$-portal right-handed neutrino dark matter in the minimal $\mathrm{U}(1)_{X}$ extended Standard Model, Phys. Rev. D 95, 035025 (2017).

[86] https://github.com/restrepo/DiracMajorana.

[87] S. Patra, W. Rodejohann, and C. E. Yaguna, A new $B-L$ model without right-handed neutrinos, J. High Energy Phys. 09 (2016) 076. 